\title{
Counseling for health behavior change in people with COPD: systematic review
}

\author{
This article was published in the following Dove Press journal: \\ International Journal of COPD \\ 26 July 2017 \\ Number of times this article has been viewed
}

\section{Marie T Williams' \\ Tanja W Effing ${ }^{2,3}$ \\ Catherine Paquet ${ }^{4}$ \\ Carole A Gibbs ${ }^{5}$ \\ Hayley Lewthwaite' \\ Lok Sze Katrina $\mathrm{Li}^{6}$ \\ Anna C Phillips ${ }^{6}$ \\ Kylie N Johnston ${ }^{6}$}

'Health and Alliance for Research in Exercise, Nutrition and Activity (ARENA), School of Health Sciences, Sansom Institute for Health Research, University of South Australia, ${ }^{2}$ Department of Respiratory Medicine, Repatriation General Hospital, ${ }^{3}$ School of Medicine, Flinders University, ${ }^{4}$ Division of Health Sciences, Centre for Population Health Research, School of Health Sciences, Sansom Institute for Health Research, ${ }^{5}$ Library, University of South Australia, ${ }^{6}$ Division of Health Sciences, School of Health Sciences, Sansom Institute for Health Research, University of South Australia,

Adelaide, SA, Australia
Correspondence: Marie T Williams Health and Alliance for Research in Exercise, Nutrition and Activity (ARENA), School of Health Sciences, Sansom Institute for Health Research, University of South Australia, North Terrace, GPO Box 247I, Adelaide, SA 5000, Australia

Emailmarie.williams@unisa.edu.au
Abstract: Counseling has been suggested as a promising approach for facilitating changes in health behavior. The aim of this systematic review of counseling interventions for people with COPD was to describe: 1) counseling definitions, 2) targeted health behaviors, 3 ) counseling techniques and 4) whether commonalities in counseling techniques were associated with improved health behaviors. Ten databases were searched for original randomized controlled trials which included adults with COPD, used the term "counseling" as a sole or component of a multifaceted intervention and were published in the previous 10 years. Data extraction, study appraisal and coding for behavior change techniques (BCTs) were completed by two independent reviewers. Data were synthesized descriptively, with meta-analysis conducted where possible. Of the 182 studies reviewed as full-text, 22 were included. A single study provided a definition for counseling. Two key behaviors were the main foci of counseling: physical activity $(n=9)$ and smoking cessation $(n=8)$. Six studies (27\%) reported underlying models and/or theoretical frameworks. Counseling was the sole intervention in 10 studies and part of a multicomponent intervention in 12. Interventions targeting physical activity included a mean of $6.3( \pm 3.1)$ BCTs, smoking cessation 4.9 $( \pm 2.9)$ BCTs and other behaviors $6.5( \pm 3.9)$ BCTs. The most frequent BCTs were social support unspecified $(n=22 ; 100 \%)$, goal setting behavior $(n=11)$, problem-solving $(n=11)$ and instructions on how to perform the behavior $(n=10)$. No studies shared identical BCT profiles. Counseling had a significant positive effect for smoking cessation and positive but not significant effect for physical activity. Counseling for health behavior change was rarely defined and effectiveness varied by target behavior. Provision of specific details when reporting studies of counseling interventions (definition, BCTs, dosage) would allow clarification of the effectiveness of counseling as an approach to health behavior change in people with COPD.

Keywords: health behavior counseling, COPD, behavior change techniques

\section{Introduction}

Modification of health-related behaviors is associated with potential health gains but presents considerable challenges for people with chronic conditions such as COPD. For people with COPD, modifiable health-related behaviors include smoking, diet and nutrition, activity profiles, sleep quality, medication adherence and socialization. For most of these health behaviors, there is at least one recent comprehensive, systematic review exploring components and combinations of interventions directed toward changing health behaviors and their related factors in people with COPD: physical activity, ${ }^{1-5}$ self-management, ${ }^{6}$ anxiety and depression, ${ }^{7}$ smoking cessation, ${ }^{8,9}$ role of caregivers ${ }^{10}$ and intervention adherence. ${ }^{11}$ 
Counseling for specific target behaviors has been suggested for inclusion within complex interventions as a means of increasing and maintaining beneficial behavior changes. ${ }^{2,4,12,13}$ Counseling in this context is directed toward facilitating specific behavior(s). However, in line with the term "health coaching," there is no universally accepted definition for counseling. ${ }^{14,15}$ Depending on the professional discipline, context and intent, a range of terms may be used to describe specific types or strategies/techniques used with this process (i.e. counseling, coaching, advising, mentoring, cognitive behavioral therapy $[\mathrm{CBT}]$ and motivational interviewing ${ }^{16,17}$ ).

In an established taxonomy of behavior change techniques (BCTs), ${ }^{18}$ counseling directed toward change in a specific behavior is listed in the coding definition of "social support unspecified," a category that also includes CBT and motivational interviewing. Within the context of COPD management, it is likely that counseling refers to a process based on a range of theoretical frameworks and includes multiple BCTs. The aim of this systematic review was to describe and explore health counseling interventions for people with COPD. The four primary objectives of this review were to describe:

1. How counseling has been defined.

2. The range of health behaviors for which counseling approaches have been investigated.

3. Counseling techniques used for changing targeted health behaviors.

4. Where possible, commonalities in counseling techniques associated with significant improvements (effect sizes) in similar health behaviors.

A secondary objective of this review was to describe the frequency of reporting of uptake, withdrawal and adherence rates for counseling interventions targeting health behavior change in people with COPD.

\section{Methods}

The search strategy and review protocol were registered prospectively with the International Prospective Register of Systematic Reviews (PROSPERO 2016:CRD42016046415). A comprehensive database search was undertaken using AMED (Allied and Complementary Medicine), CINAHL, Cochrane Library, CENTRAL, EMBASE, Medline, Medline: epub ahead of print, PsycINFO, Scopus and Web of Science. The search strategy was led and performed by an academic librarian (CG), ${ }^{19}$ with the final search of all databases undertaken during a single week. Search terms and associated "mesh" alternatives adapted for use in all databases included the population (COPD) and intervention of interest (health counseling). The search was limited to studies published in the past 10 years (January 2006 to August 26, 2016) to reflect current clinical practice (example search strategy is provided in Table S1).

Rather than defining "health behavior counseling" a priori, that is, deciding which processes or forms of counseling should be included, we opted to take a much more pragmatic approach. Studies were eligible for inclusion in this review if authors reported the term "counseling" when describing the health behavior intervention. In addition, studies were eligible for inclusion if: 1) the full original primary study was published in English and included adults with COPD with a physician or spirometric diagnosis of COPD or COPD/ asthma overlap irrespective of disease severity or comorbidities; 2) the study design was a randomized controlled trial (RCT);3) the intervention included counseling (individual or group) either as a component of a multifaceted intervention or as a single stand-alone intervention irrespective of setting and 4) the study included any form of comparator group (e.g. true control conditions, usual care, other forms of counseling, pharmacological, complimentary therapies, exercise and other therapies). Reference lists of included studies and systematic reviews identified during the search were reviewed to identify additional potentially relevant studies.

Studies were excluded if: 1) participants were not adults, had a primary pulmonary condition other than COPD or COPD data were not reported separately to other chronic conditions; 2) the psychological or behavioral intervention concerned specific forms of CBT, genetic counseling or end-of-life planning; 3) secondary analysis of primary study data was undertaken for cost-benefit analysis or to report associations between physiological and behavioral responses in participants and 4) the citations were abstracts, study protocols, commentaries or narrative reviews.

Titles and abstracts of studies identified from the search were screened against a priori eligibility criteria independently by two reviewers (CG, MTW). Where a study could not confidently be excluded, the study was retained for fulltext screening. Studies retained for full-text screening were reviewed by two independent reviewers (CG, MTW) until consensus was reached. Where full-text papers reported secondary analysis of an RCT, the original primary study was sought.

A data extraction template was developed prospectively, pilot tested on $10 \%$ of randomly selected studies eligible for inclusion and refined accordingly. Two reviewers independently extracted all data from each included study with 
disagreements in data collection resolved by discussion and consensus. Data extracted from eligible studies included:

- Publication demographics: year of publication, country of origin (based on the study location or first author's affiliation), study design.

- Participants: group mean and SD for age, pulmonary function (ie, forced expiratory volume in 1 second [FEV ${ }_{1}$, ratio of $\mathrm{FEV}_{1}$ to forced vital capacity [FVC]), method of diagnosis (self-report, physician diagnosis, spirometry), sample size.

- Intervention: details of counseling intervention(s), ie, reported definition, target behavior, format (individual versus group sessions), duration (of each session, length of program, ie, number of weeks/sessions), BCTs ${ }^{18,20}$ and theoretical framework.

- Comparator: details of intervention(s) for the "control" condition or comparator, ie, usual care, placebo/sham "intervention," wait-listed, exercise therapy, education.

- Outcomes: data for the primary outcomes of interest for this review were extracted verbatim for counseling definitions (Objective 1), targeted health behaviors (Objective 2), counseling techniques/approaches (Objective 3), sample size and within- and between-group differences for the stated primary outcomes per study for the final assessment point in the study to calculate effect sizes (Objective 4). Data were extracted for participant uptake, withdrawal and counseling session adherence rates (secondary outcomes of interest for this review). Where outcome data were presented predominately in figures, online software ${ }^{21}$ was used and where appropriate, extracted data were cross-checked and compared with Lahham et al. ${ }^{2}$

\section{Data management and synthesis Primary objectives}

The risk of methodological bias was assessed and reported using an instrument designed for RCTs (Physiotherapy Evidence Base scale for RCTs, PEDro). ${ }^{22}$ Descriptive summaries of data extracted from each study were created and used to calculate the proportion of studies reporting a definition for counseling and describe the range of targeted health behaviors. To characterize the counseling interventions in terms of BCTs, the BCT taxonomy v1 (BCTT v1) established by Michie et al ${ }^{18,20}$ was used. The BCTT v1 is an extensive taxonomy of 93 consensus-agreed, distinct BCTs with labels and definitions. ${ }^{18}$ Two team members (KJ, TE) trained in coding for the BCTT $\mathrm{v}^{23}$ independently reviewed and coded all interventions within included studies with disagreements resolved by consensus. The number and type of BCTs for each study were calculated. Tables and figures include only the BCTT v1 labels that were identified in the included studies; thus, not all 93 of the possible BCTs are reported in this review if a BCT was not coded in any of the studies.

Separate meta-analyses were planned for target behaviors where there was sufficient commonality between BCTs; the same outcome measure was reported in more than a single study and the comparator group did not include counseling. Where exact mean and SD were not reported, these were estimated using procedures recommended by the Cochrane Collaboration. ${ }^{24}$ When the control/comparator group was used for multiple interventions, the sample size of the control/ comparator group was divided by two to avoid double counting. Chance of publication bias was planned to be assessed by visually examining asymmetry in funnel plots and tests for asymmetry if 10 studies or more were available. ${ }^{24-26}$ Meta-analyses using fixed-effects model, where appropriate, were performed using MedCalc Statistical Software version 17.0.4. ${ }^{27}$ Heterogeneity was assessed using $I^{2}$ index and the $Q$ statistic.

\section{Secondary objectives}

Uptake into the study was determined as the number of participants enrolled into the study as a percentage of those screened for suitability. Withdrawal rates were calculated for intervention and control groups separately, as the percentage of those who did not complete the study after enrolling into the intervention or control groups, respectively. Where two intervention groups (both including counseling) or two control groups existed in the same study, an average rate was reported.

\section{Results}

The outcome of the search strategy and decisions concerning inclusion/exclusion are presented in Figure 1. Of the 182 studies reviewed as full-text, 22 studies were eligible for inclusion in this review. A summary of included studies is presented in Table 1. Overall methodological quality ranged from fair to high (PEDro scores: high, $n=9$ (41\%); fair, $n=11$ (50\%); poor, $\mathrm{n}=2(9 \%)$; $^{28}$ Table $\left.\mathrm{S} 2\right)$.

Across all included studies, the average age of participants was 62 years (mean age range 51-70 years), with a mean $\mathrm{FEV}_{1} \%$ predicted ranging from $38 \%$ to $68 \%$ (excluding the study by Yuan et $\mathrm{al}^{29}$ where $\mathrm{FEV}_{1} \%$ predicted was $91 \%$ at entry to a 18-year follow-up of smoking cessation and COPD prevalence). The proportion of females within studies ranged from $4 \%$ to $55 \%$. With the exception of the study by Hilberink et al, ${ }^{30}$ all studies used spirometry to confirm a diagnosis of COPD. Counseling was provided by a range of 


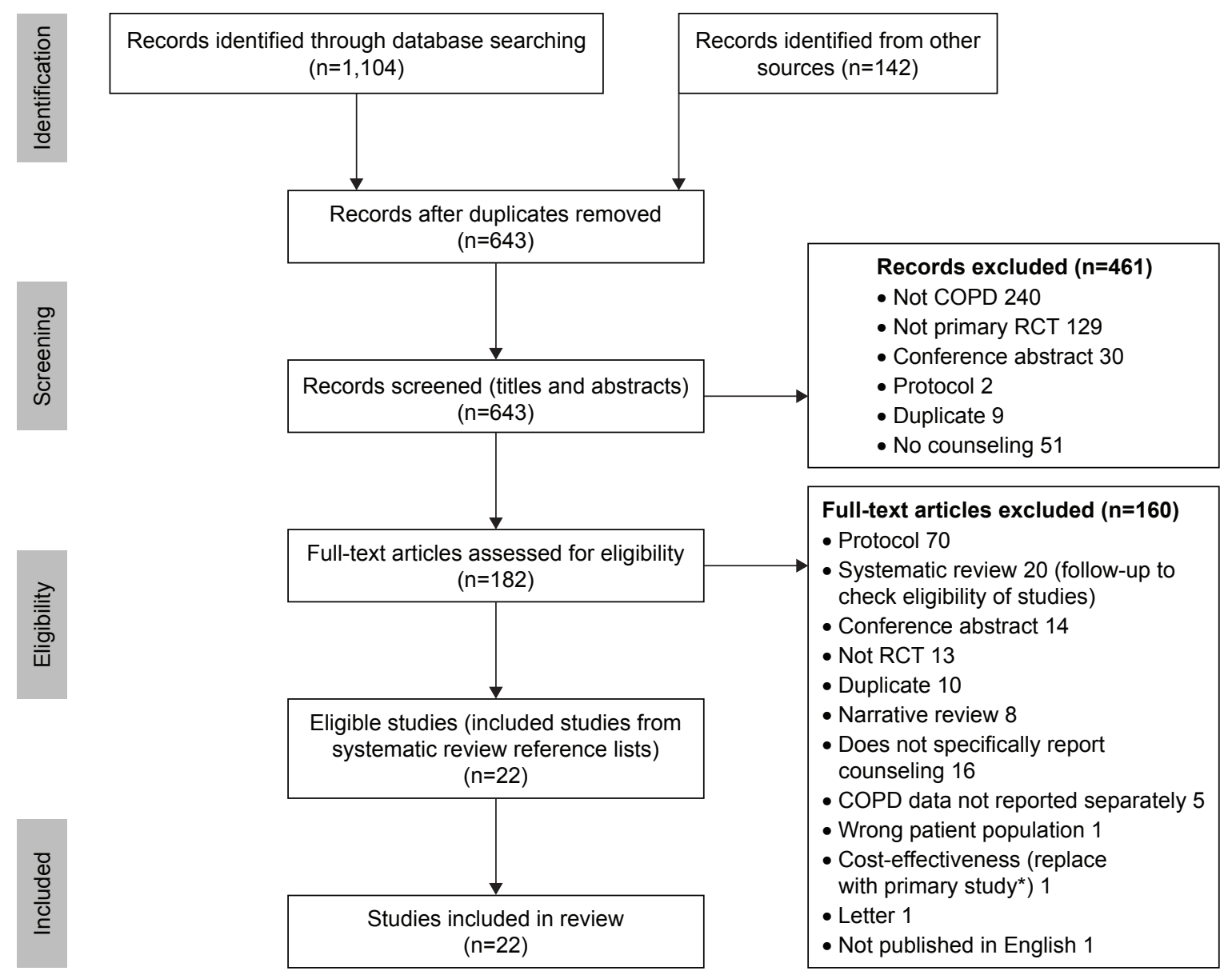

Figure I Summary of search strategy outcome.

Note: *Secondary analysis replaced with original study resulted in one paper (Wagena et al ${ }^{40}$ ) preceding publication time frame (2006-20I6). Abbreviation: RCT, randomized controlled trial.

health care practitioners with varying levels of training in counseling or BCTs (Table 1).

\section{Objective I: definitions of counseling}

While the majority of included studies provided a description of the activities included within the counseling component of the intervention ( $\mathrm{n}=14,64 \%)$, only one provided a definition for counseling: Kotz et al, ${ }^{31}$

Confrontational counselling is a patient-centred approach that involves confronting smokers with the consequences of their addiction (previously undiagnosed COPD) and which uses specific communication skills to identify and challenge irrational beliefs about smoking.

\section{Objective 2: range of health behaviors for which counseling approaches were investigated}

Two key behaviors were the foci of counseling for the majority of the included studies: physical activity $(n=9)$ or smoking cessation ( $\mathrm{n}=8$; Table 1$)$. The counseling focus for the remaining four studies was stated as being selfmanagement $(n=1)$, stress management $(n=1)$, medication adherence $(\mathrm{n}=1)$ and dietary energy intake $(\mathrm{n}=1)$. One study did not report the counseling focus. ${ }^{32}$ This study explored the addition of osteopathic manipulation to comprehensive pulmonary rehabilitation (PR), where psychological counseling was included as part of standard comprehensive PR common to both the intervention and comparator groups. As the outcomes concerned function/physical activity, this study ${ }^{32}$ was grouped with those exploring physical activity.

\section{Objective 3: counseling techniques/ approaches used for changing targeted health behaviors}

Out of 22 studies, six (27\%) reported that their intervention was based on one $(n=2)$ or more $(n=4)$ models and/or theoretical frameworks (Table 1). Three studies appeared to use the same intervention (and theoretical framework) with Altenburg et $\mathrm{al}^{33}$ referring to Hospes et $\mathrm{al}^{34}$ and de Blok et a $\mathrm{l}^{35}$ 


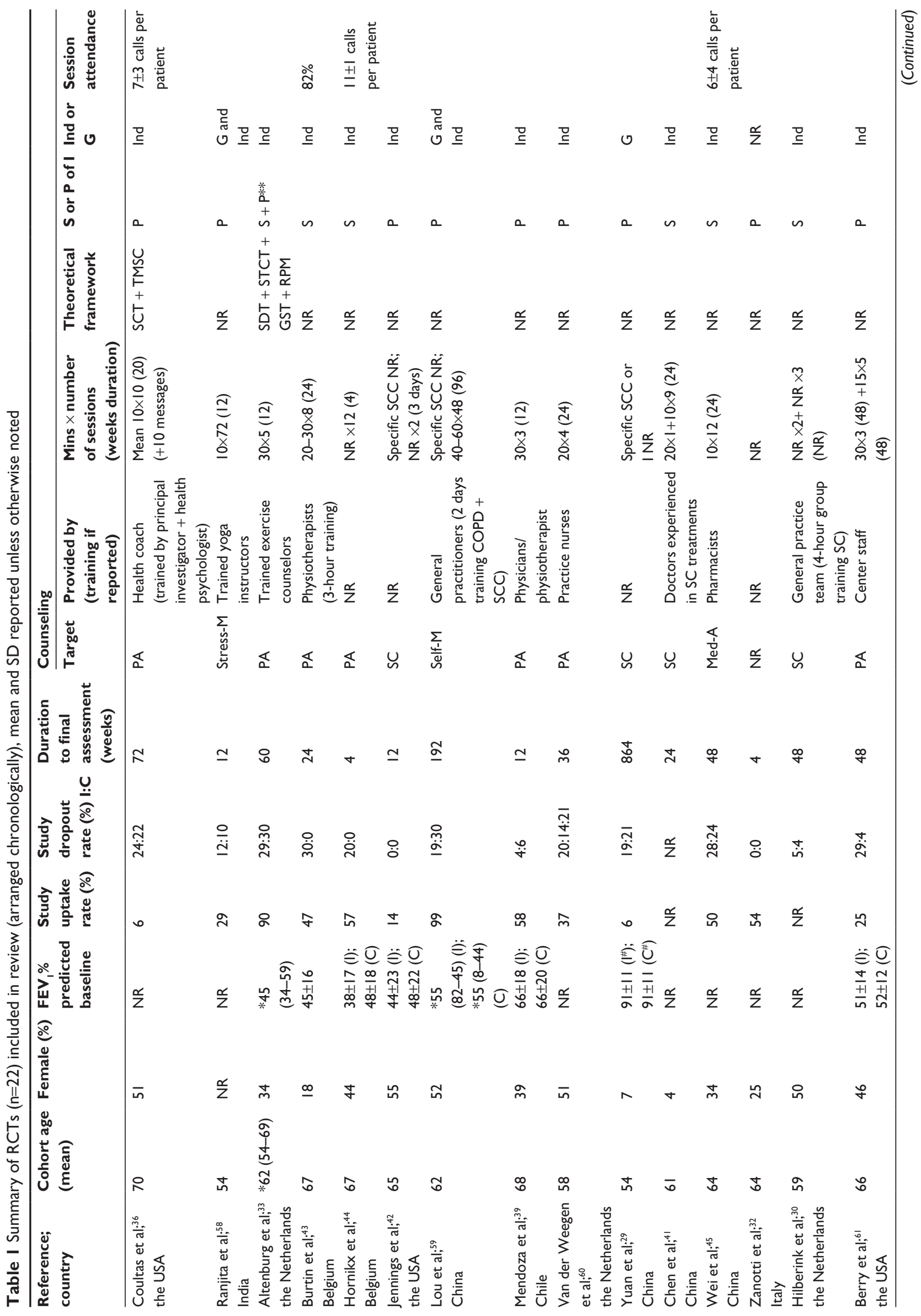




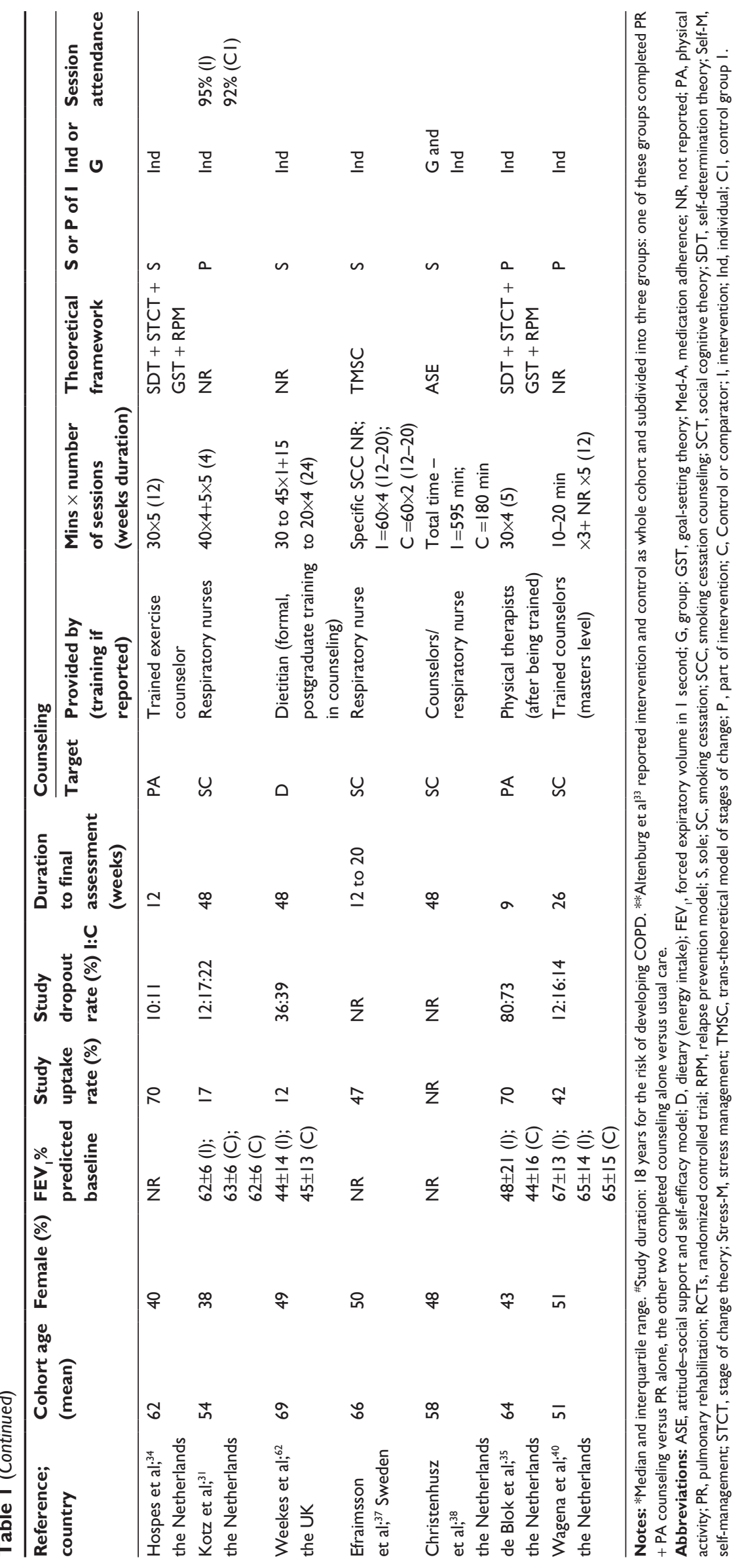


for details concerning the intervention. In these three studies, components within the intervention were based upon three theories and one model (self-determination theory, stage of change theory, goal-setting theory and the relapse prevention model). Two studies reported using the "trans-theoretical model of stages of change" $" 36,37$ with single studies reporting the use of the "attitude-social support and self-efficacy" model ${ }^{38}$ and "social cognitive theory." 36

Counseling was the sole intervention provided within intervention groups in 10 of the $22(45 \%)$ included studies (Table 1). In the remaining $12(55 \%)$ studies, counseling was part of a multicomponent intervention (eg, pedometer use, comprehensive PR and pharmacotherapy; Table S3A-C). In three studies, participants in the control/comparison group also received counseling as a component of care..$^{32,39,40}$

In general, the information provided in studies was not detailed enough to define separate BCTs delivered as a direct part of "counseling" within multicomponent interventions. BCTs were included in the description of all intervention groups ( $n=23$ ) and in five (out of 24) control groups of the 22 included studies. Details of the BCTs included in each study are presented in Table S4. Frequencies of all BCTs described in interventions to promote physical activity, smoking cessation and other health behaviors are shown in Figure 2. On average, interventions targeting physical activity reported $6.3( \pm 3.1)$ (mean $[\mathrm{SD}]$ ) different BCTs and interventions targeting smoking cessation included $4.9( \pm 2.9)$ BCTs. Studies targeting other behaviors included an average of $6.5( \pm 3.9)$
BCTs. In contrast, control groups reported few or no BCTs for all target behaviors (Table 2).

The most frequent BCTs described in study interventions were "social support (unspecified)" in 22 (100\%) studies: "goal setting (behavior)" and "problem solving" each in $11(50 \%)$ studies and "instructions on how to perform the behavior" in $10(45 \%)$ studies.

BCTs differed between studies targeting specific health behaviors. When "social support (unspecified)" was omitted (common to all 22 studies), the five BCTs most frequently reported within studies for physical activity counseling $(\mathrm{n}=10)$ were "goal-setting behavior" and "review behavioral goals" (same studies, $\mathrm{n}=6$ ), "self-monitoring of behavior" $(n=6)$, "instructions on how to perform the behavior" $(n=4)$ and "adding objects to the environment" $(n=5)$. The five BCTs most frequently reported within studies for smoking cessation counseling $(n=9)$ were "problem-solving" $(\mathrm{n}=5)$, "pharmacological support" $(\mathrm{n}=5)$, "action planning" $(n=3)$, "instructions on how to perform the behavior" $(n=3)$ and "information about health consequences" $(n=3)$.

\section{Objective 4: commonalities in counseling techniques associated with significant improvements (effect sizes) in similar health behaviors}

There were few commonalities for the specific type and combinations of BCTs (BCT profile) included within the counseling interventions across included studies. While the

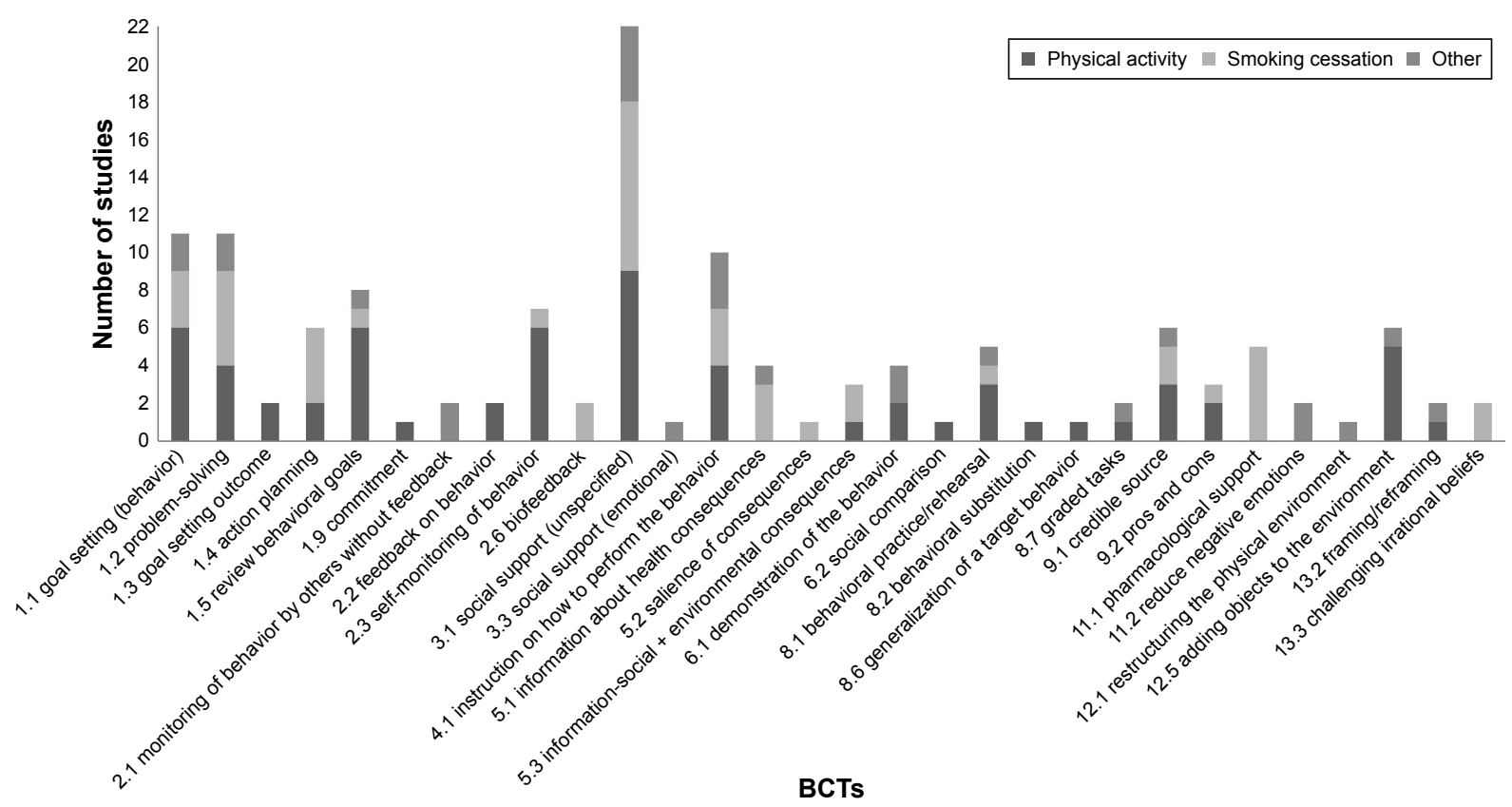

Figure 2 Frequency of BCTs described in interventions for studies included within this review. Abbreviation: BCTs, behavior change techniques. 
Table 2 Frequency of BCTs described in intervention and control/comparison groups of included studies

\begin{tabular}{|c|c|c|c|}
\hline Reference & $\begin{array}{l}\text { Counseling the } \\
\text { sole intervention? }\end{array}$ & $\begin{array}{l}\text { Total BCTs in the } \\
\text { intervention group }\end{array}$ & $\begin{array}{l}\text { Total BCTs in } \\
\text { the control group }\end{array}$ \\
\hline \multicolumn{4}{|l|}{ Physical activity } \\
\hline Coultas et $\mathrm{al}^{36}$ & $\mathrm{~N}$ & 10 & 0 \\
\hline Altenburg et $\mathrm{a}^{33}$ & $\mathrm{Y}$ & 5 & 0 \\
\hline Burtin et $\mathrm{a}^{43}$ & Y & 4 & 0 \\
\hline Hornikx et $\mathrm{al}^{44}$ & $Y$ & 8 & 0 \\
\hline Mendoza et al ${ }^{39}$ & $\mathrm{~N}$ & 4 & 4 \\
\hline Van der Weegen et a $\left.\right|^{60}$ & $\mathrm{~N}$ & 7 & 0 \\
\hline Zanotti et $\mathrm{al}^{32}$ & $\mathrm{~N}$ & I & 0 \\
\hline Berry et $\mathrm{al}^{61}$ & $\mathrm{~N}$ & 11 & 0 \\
\hline Hospes et $\mathrm{al}^{34}$ & $\mathrm{Y}$ & 5 & 1 \\
\hline de Blok et $\mathrm{al}^{35}$ & $\mathrm{~N}$ & 8 & 0 \\
\hline Mean no of BCTs $(n=10)$ & & 6.3 & 0.5 \\
\hline SD & & 3.1 & 1.3 \\
\hline Median (interquartile range) & & $6.0(5.0)$ & $0.0(0.0)$ \\
\hline \multicolumn{4}{|l|}{ Smoking cessation } \\
\hline Jennings et $\mathrm{al}^{42}$ & $\mathrm{~N}$ & 3 & 0 \\
\hline Yuan et $\mathrm{al}^{29}$ & $\mathrm{~N}$ & 5 & 0 \\
\hline Chen et $\mathrm{al}^{41}$ & $\mathrm{Y}$ & 6 & 0 \\
\hline Hilberink et al ${ }^{30}$ & $\mathrm{Y}$ & $3^{\#}$ & 0 \\
\hline Kotz et $\mathrm{a}^{31}$ & $\mathrm{~N}$ & 11 & 8 \\
\hline Efraimsson et $\mathrm{a}^{37}$ & $\mathrm{Y}$ & 6 & 0 \\
\hline Christenhusz et $\mathrm{al}^{38}$ & Y & 2 & I \\
\hline Wagena et $\mathrm{al}^{40}$ & $\mathrm{~N}$ & 3 & 2 \\
\hline Mean no of BCTs $(n=8)$ & & 4.9 & 1.4 \\
\hline SD & & 2.9 & 2.8 \\
\hline Median (interquartile range) & & $4.0(3.0)$ & $0.0(2.0)$ \\
\hline \multicolumn{4}{|l|}{ Other health behaviors } \\
\hline Lou et $\mathrm{a}^{59}$ & $\mathrm{~N}$ & 11 & 0 \\
\hline Ranjita et al ${ }^{58}$ & $\mathrm{~N}$ & 5 & 0 \\
\hline Wei et $\mathrm{al}^{45}$ & $Y$ & 2 & 0 \\
\hline Weekes et $\mathrm{al}^{62}$ & Y & 8 & 0 \\
\hline Mean no of BCTs $(n=4)$ & & 6.5 & 0.0 \\
\hline SD & & 3.9 & 0.0 \\
\hline Median (interquartile range) & & $6.5(8.0)$ & $0.0(0.0)$ \\
\hline
\end{tabular}

Note: "Only included intervention without the pharmacological component.

Abbreviations: BCTs, behavior change techniques; $\mathrm{N}$, no; $\mathrm{Y}$, yes.

BCT "social support (unspecified)" in which counseling - the term required for eligibility for this review - was common to all studies, each study presented a unique BCT profile. That is, no two studies shared identical BCT profiles for interventions targeting physical activity, smoking cessation or other behaviors. Given this variance in BCT profiles between studies and considering that the majority of studies included counseling as part of a multicomponent intervention, calculating associations between commonalities in counseling approaches and improvements in health outcomes (effect sizes) was deemed inappropriate.

Meta-analyses were undertaken to assess whether the inclusion of counseling either as a sole or part of a multicomponent intervention significantly improved health outcomes. For studies where counseling targeted physical activity, a meta-analysis was conducted pooling results across different physical activity measures with effect sizes expressed as standardized mean difference (SMD). For studies reporting on multiple outcomes, results from only one outcome were selected using the following priority ranking: 1) steps per day, 2) walking time (minutes/day), $3)$ objectively assessed moderate intensity physical activity (minute/day) and 4) 6-minute walk distance (m).

Due to the majority of studies reporting results using end point (post-intervention) values alone, or in combination with change from baseline scores, the first meta-analysis was conducted on studies reporting post-intervention values $(n=6)$ and a second on studies reporting change from baseline scores only $(n=2)$. The pooled SMD based on post-intervention values was positive, but nonsignificant 


\section{A Physical activity outcomes (post-intervention values)}

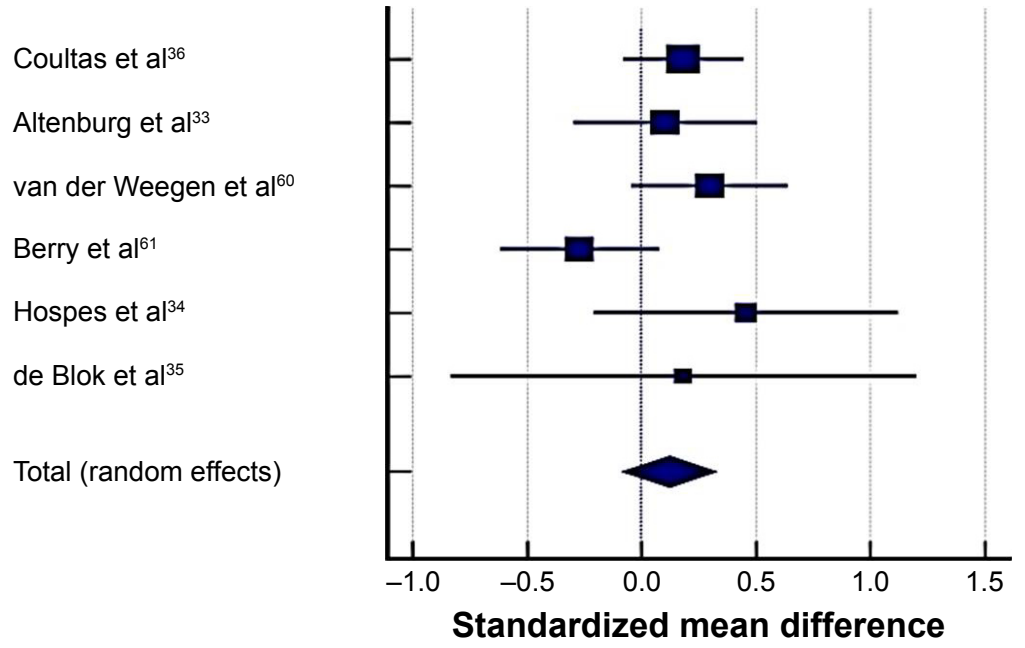

B Physical activity outcomes (Change scores)

Burtin et al ${ }^{43}$

Hornikx et al ${ }^{44}$

Total (random effects)

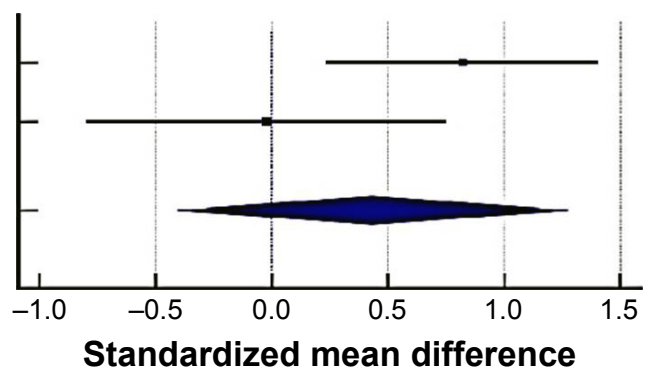

\section{Smoking cessation}

Chen et al ${ }^{41}$

Hilberink et al ${ }^{30}$

Kotz et al ${ }^{31}$ (INT1)

Kotz et al ${ }^{31}$ (INT2)

Efraimsson et $\mathrm{al}^{37}$

Christenhusz et $\mathrm{al}^{38}$

Total (fixed effects)

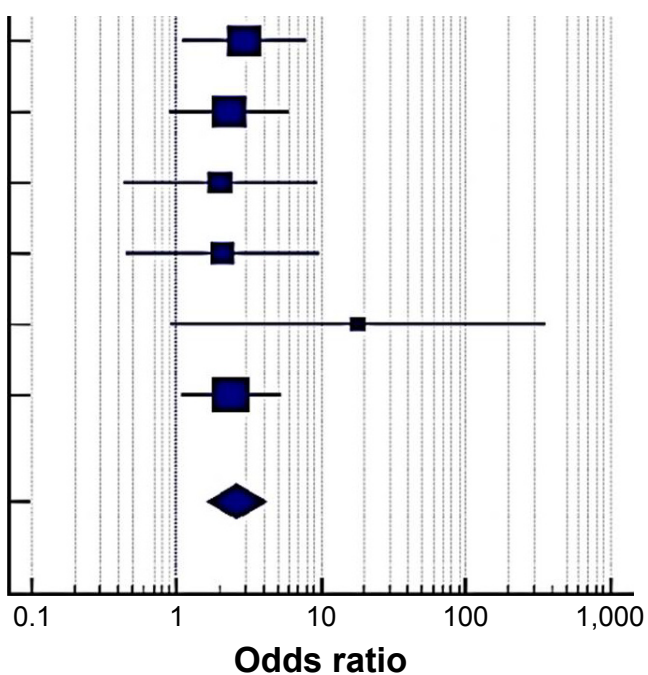

Figure 3 Individual and pooled effects of counseling interventions on physical activity outcomes: standardised mean difference for post-intervention (A) and change values (B) and smoking cessation (C).

Notes: INT I= intervention I; INT 2= intervention 2 (i.e., this study had three groups, 2 interventions and a control).

(pooled SMD [random effect] $=0.12 ; 95 \% \mathrm{CI}:-0.08$ to $0.32 ; P=0.23$; Figure $3 \mathrm{~A})$. The $I^{2}$ index and the $Q$ statistic showed moderate evidence of heterogeneity across studies $\left(I^{2}=31.41 \%\right.$ [95\% CI: 0.00, 72.20]; $\left.Q=7.29 ; P=0.20\right)$. Due to the small number of studies $(<10)$ included in the analyses, funnel plots could not be confidently interpreted. ${ }^{24,25}$ The pooled SMD for studies reporting on change only $(n=2)$ was also positive and nonsignificant (pooled SMD [random effect $]=0.43 ; 95 \% \mathrm{CI}:-0.40,1.27 ; P=0.31)$. The $I^{2}$ index and the $Q$ statistic showed some evidence of heterogeneity 
across the studies $\left(I^{2}=68.15 \%\right.$ [95\% CI: $\left.0.00,92.82\right]$; $Q=3.14 ; P=0.07$ ).

For the meta-analyses that included studies with smoking cessation as the target behavior, results from individual interventions/comparisons within studies were excluded when counseling was part of both the intervention and the control, ${ }^{31,40}$ results specific to COPD participants were not reported, ${ }^{29,41}$ nonsmokers were included ${ }^{37}$ and smoking cessation rates were not specifically reported. ${ }^{42}$ Five studies totaling six intervention groups were included in the metaanalysis with effect sizes expressed as odds ratios (Figure 3). The pooled odds ratio (fixed effect) for smoking cessation was 2.61 (95\% CI: 1.64-4.16; $P<0.001)$. There was limited evidence of heterogeneity across studies $\left(I^{2}=0.00 \%[95 \% \mathrm{CI}\right.$ : $0.00,36.8] ; Q=1.95 ; P=0.86$ ). With so few studies, funnel plots could not be confidently interpreted. ${ }^{24,25}$

\section{Secondary objectives}

Uptake (those enrolled in the study as a percentage of those screened) was reported in 19 of 22 studies (Table S5). Mean uptake into the studies was $43.6 \%$ (27) with range between $6 \%$ and $99 \%$. Withdrawal rates were very similar on average for intervention (17.2\% [SD 10.5\%]) and control groups $\left(16.9 \%\right.$ [SD 13.4\%]) and not reported in three studies. ${ }^{37,38,41}$ Participation in the counseling part of the intervention was not reported in 17 of 22 studies (Table 1). Two studies reported participation in counseling as the proportion of the planned sessions that had been delivered $\left(82 \%{ }^{43}\right.$ and $\left.95 \%{ }^{31}\right)$. Three other studies reported participation as the number of counseling sessions delivered per patient (mean 7 [SD =3] sessions; ${ }^{36} 11$ [1] sessions ${ }^{44}$ and 6 [4] sessions) ${ }^{45}$

\section{Discussion}

This systematic review identified 22 RCTs that explicitly used the term "counseling" to describe all or part of the study intervention directed toward changing a target health behavior in people with COPD. The majority of studies did not provide a definition for counseling. In these studies, counseling interventions were directed primarily toward changing behavior in physical activity and smoking cessation, incorporated an average of six specific BCTs, and within the restricted meta-analyses demonstrated significant positive effects for smoking cessation, but not for physical activity. Participation rates of study subjects in the counseling intervention was reported by the minority of studies $(n=5)$.

As part of eligibility criteria, the term "counseling" was required to be reported in the title, abstract or main body of the published study when describing the intervention. While this resulted in the exclusion of studies using accepted forms of counseling such as CBT (if they did not report the term counseling), we purposefully did not a priori define counseling as we were interested in how counseling to promote a specific behavior change in people with COPD within RCTs had been defined. In this review, only a single study provided an explicit definition. ${ }^{31}$ This relative absence of counseling definitions might have resulted from a lack of suitable health behavior counseling definitions, the nature of the studies (where counseling was a component of an intervention and not sole intervention for over half of the included studies $[n=12])$, a preference by authors to describe the theoretical framework/model or BCTs underpinning counseling as a proxy definition, or an assumption that a common understanding exists for behavioral counseling. In the broad arena of health promotion, the term "counseling" when used in the context of facilitating change in a particular health behavior appears to be used as a generic term encompassing a range of approaches. ${ }^{8,12}$ For the counseling professions, a consensus-based definition published in 2014 defined counseling as “ $\ldots$ a professional relationship that empowers diverse individuals, families, and groups to accomplish mental health, wellness, education, and career goals." ${ }^{46}$ Rather than a specific intervention, this consensus-based definition identifies a process. Depending upon the specific context and needs of the client, processes such as health counseling and/or health coaching ${ }^{15,47}$ will encompass a range of therapeutic approaches such as health contracts ${ }^{48}$ motivational interviewing ${ }^{49}$ and advice. To have greater consistency in research and clinical practice, Roche and Bourbeau ${ }^{15}$ recently proposed the need to agree upon a universal definition for health coaching and what it encompasses. Clarification of whether the terms "health coaching" and "health counseling" describe the same process and if not, how these differ, would be a useful part of this conversation.

Smoking cessation and increasing the amount of time spent in physical activity are priority behavioral targets for people with COPD, and consequently it was not surprising that these two foci comprised the majority of studies included within this review. The relatively recent availability of a taxonomy for classifying the smallest active ingredients of interventions capable of inducing change in behavior $(\mathrm{BCTs})^{18,50}$ provides an opportunity to classify intervention components $^{5}$ and potential to identify which BCTs might be associated with larger intervention effect sizes. ${ }^{51}$ Using the BCTT v1 ( $\mathrm{n}=93$ BCTs $),{ }^{18}$ the frequency and type of BCTs reported in counseling interventions included within this review differed between physical activity and smoking 
cessation, with heterogeneity between BCT profiles within studies for specific target behaviors prohibiting the identification of which specific BCTS might be associated with larger effect sizes for the counseling interventions. Two recent previous reviews of randomized/controlled studies in people with COPD have incorporated coding for BCTs as part of their methodology. While not intending to explore the association between specific BCTs and effect sizes for changes in physical activity, Wilson et $\mathrm{al}^{5}$ used a taxonomy of physical activity and healthy eating behaviors (Coventry, Aberdeen and London - Refined [CALO-RE] taxonomy) as part of a review of 20 studies (mean BCTS across studies $=6$ [range 1-13]). Bartlett et $\mathrm{al}^{9}$ reviewed 17 studies of smoking cessation interventions using a taxonomy of behavior changes specific to smoking cessation ( $n=53$ BCTs), reported a mean of 13.11 BCTs across studies (SD 8.63; range 1-28) and identified four established (facilitate action planning/develop treatment plan, prompt self-recording, advise on methods of weight control and advise on/facilitate use of social support) and one new BCT (linking COPD and smoking) that were associated with significantly larger effect sizes for quit rate. Alternatively, in reviews of behavior change interventions in populations other than people with COPD, authors have sought to examine whether incorporating a greater number of BCTs increases intervention effectiveness. For example, a review of Internet-based interventions to promote health behavior found those with more BCTs tended to have larger effect sizes compared with those that incorporated fewer techniques. ${ }^{51}$ In contrast, a review of the "active ingredients" in physical activity and diet interventions on weight loss in obese participants did not find a greater effect with more BCTs. $^{52}$

While this system and approach for identifying BCTs holds great future promise, retrospectively interrogating studies for BCT content raise a number of practical and theoretical issues. The most commonly reported limitation is the lack of reporting on specific BCTs within interventions ${ }^{11,13}$ with Sheeran et al ${ }^{53}$ noting that " ... behavioral counseling is probably the most notorious example." Issues as outlined by Sheeran et $\mathrm{al}^{53}$ include the time required for training to code discrete BCT techniques, the modest reliability for a subset of these BCTs, discrepancies between the BCTs reported in interventions and those included within the coding manuals and the need to report and code BCTs included within control/comparator conditions. Considerations that do not appear to have been addressed in previous reviews of BCTs for specific health behaviors in people with COPD (or other populations) are the potential effects of intervention dosage (intensity, duration) and participation rates (adherence), both of which might be expected to moderate BCT effect.

In this review, the difficulty of exploring the impact (effect size) of health behavior counseling approaches was confounded by a number of factors: 1) the heterogeneity of BCTs (number, type and specific target behavior) and outcome measures between studies; 2) inclusion of counseling approaches within multicomponent interventions especially where one of the strategies included a powerful behavior change agent such as supervised exercise (physical activity) or pharmacological support (smoking cessation); 3) comparator groups that include a counseling approach; 4) the lack of reporting concerning participant attendance/adherence to the counseling aspect of interventions and 5) heterogeneity in the dosage/duration of the intervention and follow-up assessment time points (immediate short-term impact of the intervention versus longer term inferred habitual lifestyle change).

Consequently, we undertook a very conservative and limited meta-analysis to compare interventions with and without counseling. The findings of these were in line with previous meta-analysis of behavioral interventions to facilitate smoking cessation ${ }^{8,9}$ but were more equivocal for physical activity counseling. This discrepancy might be simply explained by the small and selected number of studies which included the term "counseling" to describe the intervention or the use of the final assessment point for each study as opposed to the more immediate, short-term outcomes where the intervention was likely to have the greatest effect. Alternatively, it is possible that the number, type and dosage of BCTs included within studies grouped as physical activity counseling differ between reviews.

\section{Does use of the term "counseling" within interventions make a difference?}

We excluded five studies from this review solely because they did not specifically use the term "counseling" when describing an intervention targeting physical activity in people with COPD: "health contract,"48 "motivational interviewing techniques," "motivational feedback," 55 "motivational cues" $" 56$ and "motivational interview." $" 57$ Four of these studies ${ }^{48,54-56}$ have been included in previous systematic reviews of physical activity outcomes in people with COPD..$^{2-5}$

When intervention descriptions for the five excluded studies were coded for BCTs, there was a greater number of BCTs for the excluded studies $(n=5$; mean number of $\mathrm{BCTs}=10.2$; SD 2; range 8-11) compared to physical activity counseling studies included in this review $(\mathrm{n}=10$; mean 6.2 ; 


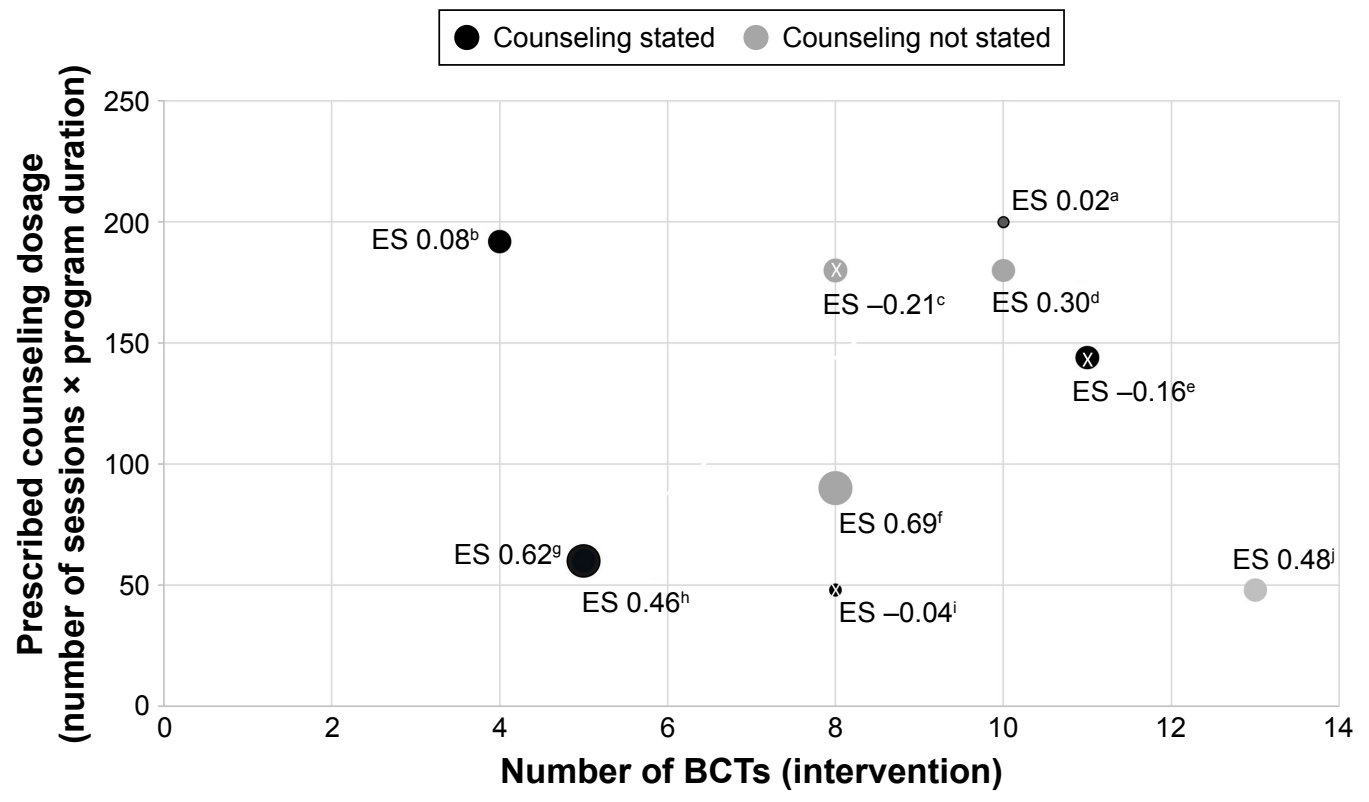

\begin{tabular}{|c|c|}
\hline \multicolumn{2}{|l|}{ Effect size $\times$ - favors control } \\
\hline${ }^{a}$ Coultas et $a^{36}$ & TTabak et al ${ }^{56}$ \\
\hline${ }^{\mathrm{b}}$ Burtin et $\mathrm{al}^{43}$ & ${ }^{9}$ Altenburg et $\mathrm{al}^{33}-3$-month assessment \\
\hline${ }^{\mathrm{C}}$ Nguyen et a ${ }^{55}$ - dosage assumes daily exposure to intervention & ${ }^{\mathrm{h}}$ Hospes et al ${ }^{34}-3$-month assessment \\
\hline dNguyen et a ${ }^{54}$ - dosage assumes daily exposure to intervention & 'Hornikx et al ${ }^{44}-1$-month assessment \\
\hline${ }^{e}$ Berry et al ${ }^{61}$ & jCruz et $\mathrm{al}^{48}$ \\
\hline
\end{tabular}

Figure 4 Relationships between prescribed intervention dosage, number of BCTs included in intervention and between-group outcome (effect size) for change in 6MWD for studies of physical activity counseling.

Note: Assessment at 6 months unless otherwise stated.

Abbreviations: $B C T s$, behavior change techniques; 6MWD, 6-minute walk distance; ES, effect size.

SD 3; range 1-11). In addition to more frequent BCTs, the BCT profile for studies using terms such as "motivational interviewing techniques" or "health contract" differed considerably to studies using the term "counseling" (Figure S1). Whether these differences in BCT profile were reflected in the effect sizes of the interventions was explored visually (Figure 4). For studies that reported sufficient data and where the control/comparator group did not report inclusion of a counseling aspect, we plotted effect size (between group) for 6-minute walk distance (6MWD; short-term impact between 1 and 6 months), volume of BCTs within the active intervention and prescribed counseling load (number of counseling sessions $\times$ duration of intervention phase [weeks]). While the data are fragmentary, there was considerable overlap between interventions which did or did not include the term "counseling" to describe the physical activity intervention (Figure 4). In addition, there did not appear to be a clear association between counseling intervention dosage, number of BCTs and magnitude of effect size between groups.

The strengths of this review include the a priori development of the comprehensive search and review approach, the independent coding of reported BCTs for each intervention and control condition by two reviewers trained in the use of this validated taxonomy. As with all reviews undertaking retrospective analysis, we were limited by reviewing studies that were not necessarily undertaken for the primary objectives posed in this review. The impact on the review findings of excluding studies published in languages other than English is unknown.

\section{Conclusion}

Counseling approaches, whether used alone or in combination, have the potential to facilitate positive changes in a range of health behaviors. In this review of RCTs, the term "counseling" was rarely defined within health behavior change interventions for people with COPD. A range of target behaviors was evidenced, with counseling for smoking cessation demonstrating clear positive effects but findings for physical activity counseling were equivocal. The identification of the active ingredients or BCTs leading to changes in target health behaviors was limited by the clarity of intervention description, consistency of behavior change interventions and provision of information concerning likely moderators of intervention effects between studies. Definitions for health 
behavior counseling (and other approaches) and taxonomies for classifying BCTs (and associated analytics) will continue to evolve. Given the number of published study protocols for current and future trials of health behavior change interventions in people with COPD, this review has implications for clinicians and researchers to improve reporting and implementation of counseling and other behavioral approaches. We recommend that consideration should be given to 1) working toward a universally acceptable definition for behavioral approaches such as health counseling/coaching; 2) explicitly reporting target behaviors and BCTs (BCT profiles) including dosage prescription; 3) using current behavior change taxonomies to inform/guide language when describing techniques and 4) documenting participation/adherence rates wherever possible. If these four considerations were achieved by research studies currently underway (and planned), future meta-analysis may be able to identify which BCTs (for whom, how and when) are associated with the most advantageous health behavior changes for people with COPD.

\section{Disclosure}

The authors report no conflicts of interest in this work.

\section{References}

1. Almagro P, Castro A. Helping COPD patients change health behavior in order to improve their quality of life. Int J Chron Obstruct Pulmon Dis. 2013;8:335-345.

2. Lahham A, McDonald CF, Holland AE. Exercise training alone or with the addition of activity counseling improves physical activity levels in COPD: a systematic review and meta-analysis of randomized controlled trials. Int J Chron Obstruct Pulmon Dis. 2016;11:3121.

3. Lundell S, Holmner Å, Rehn B, Nyberg A, Wadell K. Telehealthcare in COPD: a systematic review and meta-analysis on physical outcomes and dyspnea. Respir Med. 2015;109(1):11-26.

4. Mantoani LC, Rubio N, McKinstry B, MacNee W, Rabinovich RA. Interventions to modify physical activity in patients with COPD: a systematic review. Eur Respir J. 2016;48(1):69-81.

5. Wilson JJ, O’Neill B, Collins EG, Bradley JM. Interventions to increase physical activity in patients with COPD: a comprehensive review. COPD. 2015;12(3):339-354.

6. Zwerink M, Brusse-Keizer M, van der Valk P, et al. Self-management for patients with chronic obstructive pulmonary disease. Cochrane Database Syst Rev. 2014;19(3):CD002990.

7. Coventry PA, Bower P, Keyworth C, et al. The effect of complex interventions on depression and anxiety in chronic obstructive pulmonary disease: systematic review and meta-analysis. PLoS One. 2013;8(4):e60532.

8. van Eerd EAM, van der Meer RM, van Schayck OCP, Kotz D. Smoking cessation for people with chronic obstructive pulmonary disease. Cochrane Database Syst Rev. 2016;8:CD010744.

9. Bartlett YK, Sheeran P, Hawley MS. Effective behaviour change techniques in smoking cessation interventions for people with chronic obstructive pulmonary disease: a meta-analysis. Br J Health Psychol. 2014;19(1):181-203.

10. Bryant J, Mansfield E, Boyes AW, Waller A, Sanson-Fisher R, Regan T. Involvement of informal caregivers in supporting patients with COPD a review of intervention studies. Int J Chron Obstruct Pulmon Dis. 2016;11:1587.
11. McCullough AR, Ryan C, Macindoe C, et al. Behavior change theory, content and delivery of interventions to enhance adherence in chronic respiratory disease: a systematic review. Respir Med. 2016;116:78-84.

12. Jepson RG, Harris FM, Platt S, Tannahill C. The effectiveness of interventions to change six health behaviours: a review of reviews. $B M C$ Public Health. 2010;10(1):538.

13. Jolly K, Majothi S, Sitch AJ, et al. Self-management of health care behaviors for COPD: a systematic review and meta-analysis. Int $J$ Chron Obstruct Pulmon Dis. 2016;11:305-326.

14. Kivelä K, Elo S, Kyngäs H, Kääriäinen M. The effects of health coaching on adult patients with chronic diseases: a systematic review. Patient Educ Couns. 2014;97(2):147-157.

15. Roche N, Bourbeau J. Health coaching: another component of personalized medicine for patients with chronic obstructive pulmonary disease. Am J Respir Crit Care Med. 2016;194(6):647-649.

16. Bluckert P. The similarities and differences between coaching and therapy. Ind Commer Train. 2005;37(2):91-96.

17. Cleary M, Horsfall J. Coaching: comparisons with mentoring. Issues Ment Health Nurs. 2015;36(3):243-245.

18. Michie S, Richardson M, Johnston M, et al. The behavior change technique taxonomy (v1) of 93 hierarchically clustered techniques: building an international consensus for the reporting of behavior change interventions. Ann Behav Med. 2013;46(1):81-95.

19. Sampson M, McGowan J, Cogo E, Grimshaw J, Moher D, Lefebvre C. An evidence-based practice guideline for the peer review of electronic search strategies. J Clin Epidemiol. 2009;62(9):944-952.

20. Michie S, van Stralen MM, West R. The behaviour change wheel: a new method for characterising and designing behaviour change interventions. Implement Sci. 2011;6(1):42.

21. Ankit Rohatgi. WebPlotDigitizer [Computer Program]. Version 3.11 Austin, TX: 2017.

22. Maher CG, Sherrington C, Herbert RD, Moseley AM, Elkins M. Reliability of the PEDro scale for rating quality of randomized controlled trials. Phys Ther. 2003;83(8):713.

23. BCT Taxonomy [homepage on the Internet]. Behavior Change Technique Taxonomy (BCTT) Version 1 Online Training. 2017. Available from: http://www.bct-taxonomy.com/. Accessed October 26, 2016.

24. Higgins JP, Green S. Cochrane Handbook for Systematic Reviews of Interventions. Vol. 4. Hoboken, NJ: John Wiley \& Sons; 2011.

25. Egger M, Smith GD, Schneider M, Minder C. Bias in meta-analysis detected by a simple, graphical test. BMJ. 1997;315(7109):629-634.

26. Sterne JA, Sutton AJ, Ioannidis JP, et al. Recommendations for examining and interpreting funnel plot asymmetry in meta-analyses of randomised controlled trials. BMJ. 2011;343:d4002.

27. MedCalc Software bvba. MedCalc Statistical Software [Computer Program]. Ostend, Belgium: MedCalc Software bvba; 2016.

28. Foley NC, Teasell RW, Bhogal SK, Speechley MR. Stroke rehabilitation evidence-based review: methodology. Top Stroke Rehabil. 2003;10(1):1-7.

29. Yuan X, Tao Y, Zhao JP, et al. Long-term efficacy of a rural communitybased integrated intervention for prevention and management of chronic obstructive pulmonary disease: a cluster randomized controlled trial in China's rural areas. Braz J Med Biol Res. 2015;48(11):1023-1031.

30. Hilberink SR, Jacobs JE, Breteler MH, de Vries H, Grol RP. General practice counseling for patients with chronic obstructive pulmonary disease to quit smoking: impact after 1 year of two complex interventions. Patient Educ Couns. 2011;83(1):120-124.

31. Kotz D, Wesseling G, Huibers MJ, van Schayck OC. Efficacy of confronting smokers with airflow limitation for smoking cessation. Eur Respir J. 2009;33(4):754-762.

32. Zanotti E, Berardinelli P, Bizzarri C, et al. Osteopathic manipulative treatment effectiveness in severe chronic obstructive pulmonary disease: a pilot study. Complement Ther Med. 2012;20(1-2):16-22.

33. Altenburg WA, ten Hacken NH, Bossenbroek L, Kerstjens HA, de Greef MH, Wempe JB. Short- and long-term effects of a physical activity counselling programme in COPD: a randomized controlled trial. Respir Med. 2015;109(1):112-121. 
34. Hospes G, Bossenbroek L, Ten Hacken NH, van Hengel P, de Greef MH. Enhancement of daily physical activity increases physical fitness of outclinic COPD patients: results of an exercise counseling program. Patient Educ Couns. 2009;75(2):274-278.

35. de Blok BM, de Greef MH, ten Hacken NH, Sprenger SR, Postema K, Wempe JB. The effects of a lifestyle physical activity counseling program with feedback of a pedometer during pulmonary rehabilitation in patients with COPD: a pilot study. Patient Educ Couns. 2006;61(1): $48-55$.

36. Coultas DB, Jackson BE, Russo R, et al. A lifestyle physical activity intervention for patients with chronic obstructive pulmonary disease. A Randomized Controlled Trial. Ann Am Thorac Soc. 2016;13(5): 617-626.

37. Efraimsson EÖ, Hillervik C, Ehrenberg A. Effects of COPD self-care management education at a nurse-led primary health care clinic. Scand J Caring Sci. 2008;22(2):178-185.

38. Christenhusz L, Pieterse M, Seydel E, van der Palen J. Prospective determinants of smoking cessation in COPD patients within a high intensity or a brief counseling intervention. Patient Educ Couns. 2007; 66(2):162-166.

39. Mendoza L, Horta P, Espinoza J, et al. Pedometers to enhance physical activity in COPD: a randomised controlled trial. Eur Respir J. 2015;45(2):347-354.

40. Wagena EJ, Knipschild PG, Huibers MJH, Wouters EFM, Van Schayck CP. Efficacy of bupropion and nortriptyline for smoking cessation among people at risk for or with chronic obstructive pulmonary disease. Arch Intern Med. 2005;165(19):2286-2292.

41. Chen J, Chen Y, Chen P, Liu Z, Luo H, Cai S. Effectiveness of individual counseling for smoking cessation in smokers with chronic obstructive pulmonary disease and asymptomatic smokers. Exp Ther Med. 2014;7(3):716-720.

42. Jennings JH, Thavarajah K, Mendez MP, Eichenhorn M, Kvale P, Yessayan L. Predischarge bundle for patients with acute exacerbations of COPD to reduce readmissions and ED visits: a randomized controlled trial. Chest. 2015;147(5):1227-1234.

43. Burtin C, Langer D, van Remoortel $\mathrm{H}$, et al. Physical activity counselling during pulmonary rehabilitation in patients with COPD: a randomised controlled trial. PLoS One. 2016;11(2):e0148705. PLoS One [Electronic Resource]. 2015;10(12): 0144989.

44. Hornikx M, Demeyer H, Camillo CA, Janssens W, Troosters T. The effects of a physical activity counseling program after an exacerbation in patients with chronic obstructive pulmonary disease: a randomized controlled pilot study. BMC Pulm Med. 2015;15:136.

45. Wei L, Yang X, Li J, et al. Effect of pharmaceutical care on medication adherence and hospital admission in patients with chronic obstructive pulmonary disease (COPD): a randomized controlled study. $J$ Thorac Dis. 2014;6(6):656-662.

46. Kaplan DM, Tarvydas VM, Gladding ST. 20/20: a vision for the future of counseling: the new consensus definition of counseling. $J$ Counsel Dev. 2014;92(3):366-372.

47. Linden A, Butterworth SW, Prochaska JO. Motivational interviewingbased health coaching as a chronic care intervention. J Eval Clin Pract. 2010;16(1):166-174.

48. Cruz J, Brooks D, Marques A. Walk2Bactive: a randomised controlled trial of a physical activity-focused behavioural intervention beyond pulmonary rehabilitation in chronic obstructive pulmonary disease. Chron Respir Dis. 2016;13(1):57-66.
49. Rubak S, Sandbæk A, Lauritzen T, Christensen B. Motivational interviewing: a systematic review and meta-analysis. Br J Gen Pract. 2005; 55(513):305-312.

50. Michie S, Wood CE, Johnston M, Abraham C, Francis J, Hardeman W. Behaviour change techniques: the development and evaluation of a taxonomic method for reporting and describing behaviour change interventions (a suite of five studies involving consensus methods, randomised controlled trials and analysis of qualitative data). Health Technol Assess. 2015;19(99):1-188.

51. Webb T, Joseph J, Yardley L, Michie S. Using the internet to promote health behavior change: a systematic review and meta-analysis of the impact of theoretical basis, use of behavior change techniques, and mode of delivery on efficacy. $J$ Med Internet Res. 2010;12(1):e4.

52. Dombrowski SU, Sniehotta FF, Avenell A, Johnston M, MacLennan G, Araújo-Soares V. Identifying active ingredients in complex behavioural interventions for obese adults with obesity-related co-morbidities or additional risk factors for co-morbidities: a systematic review. Health Psychol Rev. 2012;6(1):7-32.

53. Sheeran P, Klein WM, Rothman AJ. Health behavior change: moving from observation to intervention. Annu Rev Psychol. 2017;68: 573-600.

54. Nguyen HQ, Donesky D, Reinke LF, et al. Internet-based dyspnea selfmanagement support for patients with chronic obstructive pulmonary disease. J Pain Symptom Manage. 2013;46(1):43-55.

55. Nguyen HQ, Gill DP, Wolpin S, Steele BG, Benditt JO. Pilot study of a cell phone-based exercise persistence intervention post-rehabilitation for COPD. Int J Chron Obstruct Pulmon Dis. 2009;4:301-313.

56. Tabak M, Vollenbroek-Hutten MM, van der Valk PD, van der Palen J, Hermens HJ. A telerehabilitation intervention for patients with chronic obstructive pulmonary disease: a randomized controlled pilot trial. Clin Rehabil. 2014;28(6):582-591.

57. Song HY, Yong SJ, Hur HK. Effectiveness of a brief self-care support intervention for pulmonary rehabilitation among the elderly patients with chronic obstructive pulmonary disease in Korea. Rehabil Nurs. 2014;39(3):147-156.

58. Ranjita R, Hankey A, Nagendra HR, Mohanty S. Yoga-based pulmonary rehabilitation for the management of dyspnea in coal miners with chronic obstructive pulmonary disease: A randomized controlled trial. J Ayurveda Integr Med. 2016;7(3):158-166.

59. Lou P, Chen P, Zhang P, et al. A COPD health management program in a community-based primary care setting: a randomized controlled trial. Respir Care. 2015;60(1):102-112.

60. Van der Weegen S, Verwey R, Spreeuwenberg M, Tange H, van der Weijden T, de Witte L. It's LiFe! Mobile and web-based monitoring and feedback tool embedded in primary care increases physical activity: A cluster randomized controlled trial. Journal of Medical Internet Research. 2015;17(7):e184. doi:10.2196/jmir.4579.

61. Berry MJ, Rejeski WJ, Miller ME, et al. A lifestyle activity intervention in patients with chronic obstructive pulmonary disease. Respiratory Medicine. 2010;104(6):829-839.

62. Weekes CE, Emery PW, Elia M. Dietary counselling and food fortification in stable COPD: a randomised trial. Thorax. 2009;64(4):326-331.
International Journal of COPD

\section{Publish your work in this journal}

The International Journal of COPD is an international, peer-reviewed journal of therapeutics and pharmacology focusing on concise rapid reporting of clinical studies and reviews in COPD. Special focus is given to the pathophysiological processes underlying the disease, intervention programs, patient focused education, and self management protocols.

\section{Dovepress}

This journal is indexed on PubMed Central, MedLine and CAS. The manuscript management system is completely online and includes a very quick and fair peer-review system, which is all easy to use. Visit http://www.dovepress.com/testimonials.php to read real quotes from published authors. 